\title{
Discrimination learning as a function of the duration of rewarding hypothalamic stimulation '
}

\author{
ERNEST P. LINDHOLM ${ }^{2}$ AND RICHARD E. KEESEY \\ UNIVERSITY OF WISCONSIN
}

Independent groups of rats received posterior hypothalamic stimulation of $0.5,1.0,3.0$, or 5.0 sec duration as a reward for performance in a brightness discrimination task. Acquisition of this discrimination was found to be inversely and linearly related to the duration of rewarding stimulation. It is suggested that long durations of hypothalamic stimulation, while generating strong approach tendencies, may retard learning by interfering with critical association and/or consolidation processes.

Considerable data exists relating the various electrical parameters of intracranial stimulation (ICS) to the free operant rate of self-stimulation. In contrast, ICS has only infrequently been used as a reward in discrimination learning tasks and relatively little is known concerning the effects of systematic manipulation of its electrical parameters on performance in such situations. One exception is a recent report (Keesey, 1966) in which ICS conditions differing in pulse frequency and current served as the reward in a two-choice learning problem requiring a brightness discrimination. The rate at which this discrimination was acquired was inversely related to the pulse frequency, but independent of the pulse current. It would appear, therefore, that certain electrical parameters of ICS reward may influence the acquisition of new habits as well as their free operant rate of occurrence once they have been established. In the following experiment, this suggestion is subjected to further test by investigating the influence of the ICS train duration on the acquisition of a brightness discrimination.

\section{Subjects}

Data were obtained from 48 male Sprague-Dawley rats weighing 210-250 g. Each was implanted with a bipolar stainless steel electrode in the region of the posterior hypothalamus.

Apparatus

All training and testing took place in a $\mathrm{Y}$ maze brightness discrimination apparatus which differed only in detail from the one described by Keesey (1966). Each arm of the maze contained a response lever over which was mounted a 1 in. pilot light assembly. These lamps served as the discriminanda, and a brightness difference was achieved by passing $75 \mathrm{~V}$ ac across one and $115 \mathrm{~V}$ ac across the other. The ICS consisted of a train of symmetrical biphasic pulses of constant current. The pulse duration and frequency were fixed at $0.1 \mathrm{msec}$ and 100 pulses/ sec, respectively.

\section{Procedure}

A two day pretraining procedure was initiated two days following surgery. On Day 1 pretraining, a behavioral sequence was established that required $S$ to (1) respond for ICS reward on one of the front bars beneath the cue lights, (2) pass to the rear of the maze and press a bar to set up the next trial, and (3) return again to the front bar for stimulation. On Day 2 pretraining, each $S$ was first assigned to one of the four experimental train duration conditions $(0.5 \mathrm{sec}, 1.0 \mathrm{sec}, 3.0 \mathrm{sec}$, or $5.0 \mathrm{sec})$ and then, with the current fixed at $1.2 \mathrm{~mA}$, given further experience on the shuttle sequence under these ICS duration conditions. Forced trials were employed throughout this pretraining phase to insure that each $S$ obtained equal experience with both sides of the maze and both brightness conditions.

Following this pretraining procedure, a simultaneous brightness discrimination problem was introduced. Employing a correction procedure, each response was reinforced by a $1.2 \mathrm{~mA}$ train of hypothalamic stimulation, the duration of which was determined by the experimental condition to which $S$ had been assigned. Following termination of the ICS on each trial, a 10 sec intertrial interval was imposed, $S$ being free to self-administer the next trial at any time after this period. This session was terminated after 400 such trials, the $S$ sacrificed, and its brain prepared for histological examination.

Results

On each trial, a record was taken of (1) the animal's

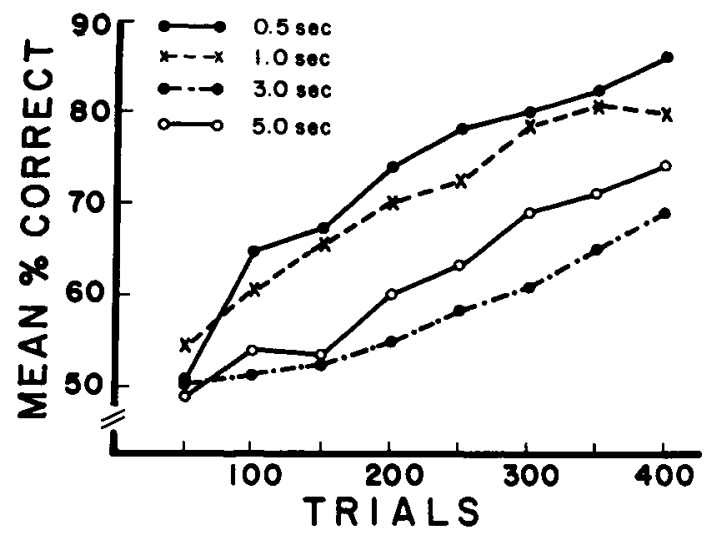

Fig. 1. Mean percent correct responses across the 400 discrimination learning trials as a function of the duration of rewarding ICS. 
brightness choice, and (2) the response latency, measured from the end of the intertrial interval to a choice. Functions relating the acquisition of the brightness discrimination to the duration of rewarding ICS are presented in Fig. 1. It is evident that increasing the ICS duration has an adverse effect on the acquisition of the brightness habit. This observation was supported by an analysis of variance which yielded a significant $F$ value for both the effect of ICS duration $(p<.025)$ and trials $(p<.01)$. In addition, an ICS duration gradient (not shown) was obtained by plotting the mean percentage correct across the entire 400 acquisition trials as a function of the reward duration. A trend analysis performed upon this function revealed only a linear trend $(p<.01)$, suggesting that acquisition is inversely and linearly related to the duration of rewarding hypothalamic stimulation.

The response latency was also influenced by the ICS duration $(p<.05)$. In agreement with the results of previous studies (Keesey, 1964; Hodos, 1965), the trend of the data was toward higher response speeds with longer durations. However, the running speeds of the 3.0 and $5.0 \mathrm{sec}$ groups tended to decline toward the end of the 400 trial session, particularly in the last 100 trial block. This produced a significant trials by duration interaction and a nonmonotonic speedduration function in the present study.

Examination of the histological material indicated that the electrodes terminated in a region roughly described by a sphere of $1 \mathrm{~mm}$ radius, including the posterior hypothalamus, medial forebrain bundle, and mammillary region. Of particular interest was the finding that Ss with electrodes in the premamillary and mammillary region, or medial posterior hypothalamus, tended to earn higher acquisition scores than Ss with placements in the medial forebrain bundle. Discussion

The present results indicate that acquisition of a brightness discrimination is an inverse function of the duration of rewarding ICS. A similar relationship has also been reported between discrimination learning and ICS pulse frequency (Keesey, 1966). Given these observations, it is clear that conclusions concerning the relative effectiveness of ICS and food as reinforcers of discrimination learning may depend on the particular set of ICS parameters chosen for the comparison. Thus, results suggesting that learning is slower when reinforced by forebrain ICS than by food (Olds, 1956) might be reconciled with a report of roughly equivalent acquisition rates with food and hypothalamic ICS reward (Kling \& Matsumiya, 1962) by a careful consideration of the differences in ICS parameters. In addition, the anatomical-behavioral relationships reported here suggest that the locus of ICS might also be critical to the effectiveness of central stimulation as a reinforcer of discrimination learning.

One way of accounting for the present results is to view longer ICS durations as having a two-fold effect, i.e., acting both to disrupt some process underlying discrimination learning while, at the same time, acting as the incentive for continued performance at the task. This disruptive effect may be analogous to that produced by posttrial electroconvulsive shock. That is, differences in discrimination acquisition might reflect differing degrees of retrograde amnesia induced by the rewarding stimulation itself. It should be noted in this regard that the posttrial delivery of low intensity electrical stimulation to certain thalamic sites has been shown to retard learning in food rewarded discrimination tasks (Mahut, $1962,1964)$. It is conceivable in the light of the present results that hypothalamic ICS used at rewarding intensities can have similar consequences.

\section{References}

HODOS, w. Motivational properties of long durations of rewarding brain stimulation. J. comp. physiol. Psychol, 1965, 59, 219-224.

KEESEY, R. E. Duration of stimulation and the reward properties of hypothalamic stimulation. J. comp. physiol. Psychol., 1964, 58, 201207.

KEESEY, R. E. Hypothalamic stimulation as a reinforcer of discrimination learning. J. comp. physiol. Psychol., 1966, 62, 231-236.

KLING, J. W., \& MATSUMIYA, Y. Relative reinforcement values of food and intracranial stimulation. Science, 1962, 135, 668-670.

MAHUT, H. Effects of subcortical electrical stimulation on learning in the rat. J. comp. physiol. Psychol., 1962, 55, 472-477.

MAHUT, H. Effects of subcortical electrical stimulation on discrimination learning in the cat.J. comp. physiol. Psychol., 1964, 58, 390-398.

OLDS, J. Runway and maze behavior controlled by basomedial forebrain stimulation in the rat. J. comp. physiol. Psychol., 1956, 49, 507-512. Note

1. This research was supported, in part, by funds supplied by the Research Committee of the University of Wisconsin Graduate School. 2. NIH Predoctoral Research Fellow. 